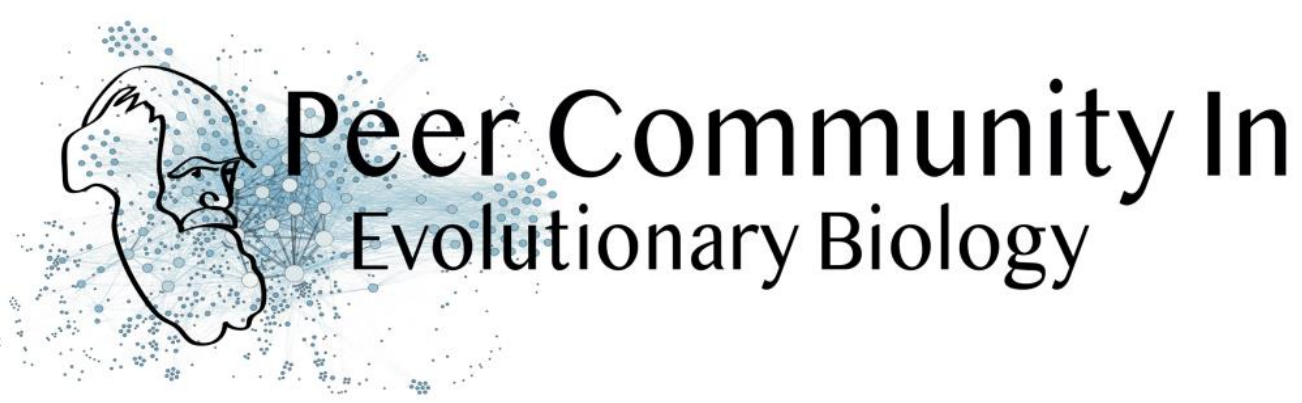

\title{
Another step towards grasping the complexity of the environmental response of traits
}

\author{
Benoit Pujol based on reviews by 2 anonymous reviewers
}

\section{A recommendation of:}

J Grey Monroe, Haoran Cai, David L Des Marais. Trait plasticity and covariance along a continuous soil moisture gradient (2021), bioRxiv, 2020.02.17.952853, ver. 5 peerreviewed and recommended by Peer Community in Evolutionary Biology. 10.1101/2020.02.17.952853

\section{Open Access}

Submitted: 20 February 2020, Recommended: 17 January 2021

\section{Cite this recommendation as:}

Benoit Pujol (2021) Another step towards grasping the complexity of the environmental response of traits. Peer Community in Evolutionary Biology, 100119. 10.24072/pci.evolbiol.100119

Published: 18 January 2021

Copyright: This work is licensed under the Creative Commons Attribution-NoDerivatives 4.0 International License. To view a copy of this license, visit http://creativecommons.org/licen ses/by-nd/4.0/
One can only hope that one day, we will be able to evaluate how the ecological complexity surrounding natural populations affects their ability to adapt. This is more like a long term quest than a simple scientific aim. Many steps are heading in the right direction. This paper by Monroe and colleagues (2021) is one of them.

Many ecological and genetic mechanisms shape the evolutionary potential of phenotypic trait variation and many of them involve environmental heterogeneity (Pujol et al 2018). To date, we cannot look into these ecological and genetic mechanisms without oversimplifying their effects. We often look into trait variation one trait at a time albeit the variation of multiple phenotypic traits is often linked at the genetic or environmental level. As a consequence, we put our conclusions at risk by not accounting for the reciprocal impacts of trait changes upon each other (Teplitsky et al 2014). We also usually restrict the study of a continuous gradient of environmental conditions to a few conditions because it would otherwise be impossible to model its environmental effect. As a consequence, we miss the full picture of the continuous often nonlinear phenotypic plastic response. Whether the trait undergo threshold effect changes thereby remains obscured to us. Collectively, these issues impede our ability to understand how selection shapes the ecological strategy of organisms under variable environments.

In this paper, Monroe and colleagues (2021) propose an original approach that raised to these two challenges. They analysed phenotypic plastic changes in response to a continuous environment in a multidimensional trait space, namely the response of Brachypodium plant developmental and physiological traits to a continuous gradient of soil moisture. They used dry down experimental treatments to produce the continuous soil moisture gradient and compared the plant capacity to use water between annual $B$. distachyon and perennial B. sylvaticum. Their results revealed the best mathematical 


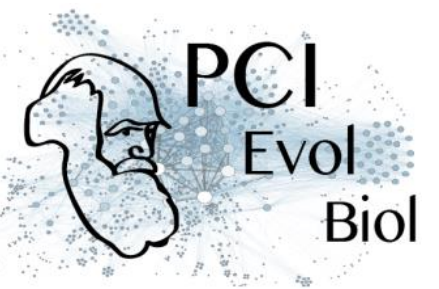

functions that model the nonlinear curvature of the continuous plastic response of Brachypodium plants. This work reinforces our view that nonlinear plastic responses can result in greater or lesser trait values at any stage of the environmental gradient that were unexpected on the basis of linear predictors (Gienapp and Brommer 2014). Their findings also imply that different threshold responses characterize different genotypes. These could otherwise have been missed by a classical approach. By shedding light on unforeseen interactions between traits that make their correlation vary along the nonlinear response, they were able to describe more accurately Brachypodium ecological strategies and the changes in evolutionary constraints along the soil moisture gradient.

Their empirical approach allows to test what environmental conditions maximises the opportunity for selection to shape trait variation. For example, it revealed unforeseen divergence in potentially adaptive mechanisms or life history strategies - and not just trait values - between annual and perennial species of Brachypodium. Behind every environmental variation of the constraints to the future evolutionary change of multiple traits, we can expect that the evolutionary history of the populations shaped their trait genetic correlations. Investigating the nonlinear signature of adaptive evolution across continuous environments will get us into uncharted territory.

Our ability to predict the adaptive potential of species is limited. With their approach of continuous environmental gradients beyond linearity, Monroe and collaborators (2021) improve our understanding of plant phenotypic responses and open a brand new range of exciting developments. As they mention: "the opportunity for scaling up" their approach is big. To illustrate this prospect, I can easily think of an example: the quantitative genetic random regression model. This model allows to use any degree of genetic relatedness in a wild population to estimate the genetic variation of phenotypic plastic reaction norms (Nussey et al 2007, Pujol and Galaud 2013). However, in this approach, only a few modalities of the environmental gradient are used to model nonlinear phenotypic plastic responses. From there, it is rather intuitive. Combining the best of these two approaches (continuity of genetic relatedness in the wild \& continuity of environmental gradient in experiments) could open ground breaking new perspectives in research.

\section{References}

Gienapp P. \& J.E. Brommer. 2014. Evolutionary dynamics in response to climate change. In: Charmentier A, Garant D, Kruuk LEB, editors. Quantitative genetics in the wild. Oxford: Oxford University Press, Oxford. pp. 254-273. doi: https://doi.org/10.1093/acprof:oso/9780199674237.003.0015

Monroe, J. G., Cai, H., and Des Marais, D. L. (2020). Trait plasticity and covariance along a continuous soil moisture gradient. bioRxiv, 2020.02.17.952853, ver. 5 peer-reviewed and recommended by $\mathrm{PCI}$ Evol Biol. doi: https://doi.org/10.1101/2020.02.17.952853

Pujol et al. (2018). The missing response to selection in the wild. Trends in ecology \& evolution, 33(5), 337346. doi: https://doi.org/10.1016/j.tree.2018.02.007

Pujol, B., and Galaud, J. P. (2013). A practical guide to quantifying the effect of genes underlying adaptation in a mixed genomics and evolutionary ecology approach. Botany Letters, 160(3-4), 197-204.

doi: https://doi.org/10.1080/12538078.2013.799045

Nussey, D. H., Wilson, A. J., and Brommer, J. E. (2007). The evolutionary ecology of individual phenotypic plasticity in wild populations. Journal of evolutionary biology, 20(3), 831-844.

doi: https://doi.org/10.1111/j.1420-9101.2007.01300.x

Teplitsky et al. (2014). Assessing multivariate constraints to evolution across ten long-term avian studies. PLoS One, 9(3), e90444. doi: https://doi.org/10.1371/journal.pone.0090444

\section{Revision round \#2}




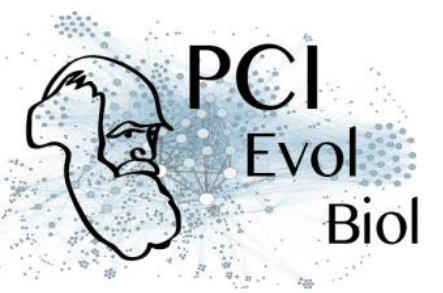

Dear authors,

the reviewers found your revision had addressed their comments. I think your paper deserves to be recommended. I neverthelless would ask a last very minor revision. Please incorporate the last few revisions required by the reviewers. I will very likely be able to assess your revised preprint without sending it out for further review this time, which should be fast. Looking forward to receiving your revision (I guess rapidly since there is not that much to do) and writting the recommendation (conditional to your revision of the preprint).

Regards, Benoit Pujol

REF1 : I really appreciate this manuscrit; I found it interesting and inspirational. The authors answered perfectly well to all my concerns. I only have one last comment, please make sure that all the figures and tables in the appendix are referenced in the main text (e.g. Fig S2, Table S2).

REF 2 : This is an updated/corrected version of a manuscript I previously reviewed. I think the authors have made a huge effort to keep the narrative of the study (more) focused, and made the statistical and biological interpretations easier to follow for the readers. The experiment the authors set up is super elegant to test a multivariate response (plasticity and evolutionary) to environmental gradients, as opposed to setting the classic anova approach. I have gone through the rebuttal letter and it appears that the authors have addressed my comments/concerns.

If anything, I would ask them to add some insights into how life-history traits and/or the ecological/environmental context in which the species evolved might influence the plastic or genetic (evolvability) response when species/genotypes encounter a "new" environment (or dimension) of their environment. Other than that, I think this is a great piece of work, the kind I would use in any evolutionary ecology/ecological genetics module.

I've added my comments in the PDF version of the manuscript. End of review

Preprint DOI: 10.1101/2020.02.17.952853

\section{Reviewed by anonymous reviewer, 2020-11-26 10:19}

I really appreciate this manuscrit; I found it interesting and inspirational. The authors answered perfectly well to all my concerns.

I only have one last comment, please make sure that all the figures and tables in the appendix are referenced in the main text (e.g. Fig S2, Table S2).

\section{Reviewed by anonymous reviewer, 2020-12-07 17:13}

This is an updated/corrected version of a manuscript I previously reviewed. I think the authors have made a huge effort to keep the narrative of the study (more) focused, and made the statistical and biological interpretations easier to follow for the readers. The experiment the authors set up is super elegant to test a multivariate response (plasticity and evolutionary) to environmental gradients, as opposed to setting the classic anova approach. I have gone through the rebuttal letter and it appears that the authors have addressed my comments/concerns. If anything, I would ask them to add some insights into how life-history traits and/or the ecological/environmental context in which the species evolved might influence the plastic or genetic (evolvability) response when species/genotypes encounter a "new" environment (or dimension) of their environment. Other than that, I think this is a great piece of work, the kind I would use in any evolutionary ecology/ecological genetics module. I've added my comments in the PDF version of the manuscript. End of review 


\title{
Revision round \#1
}

\author{
2020-06-04 \\ Dear J Grey Monroe and collaborators,
}

Thank you for submitting your preprint to $\mathrm{PCl}$ Evolutionary Biology for recommendation. Two anonymous reviewers, Rodrigo Medel (co-recommender) and I, carefully read your preprint. We found your text was elegantly written. You present an interesting perspective on the way "function-valued traits" influence the response to selection through genetic correlations and plasticity. We think that the main framework needs to be emphasized earlier in the introduction section. In its present form, most of the general context relies on particular issues related with the study system, plant variables, and soil moisture gradients. This is fine, but expanding the context/implication presented in the second paragraph throughout the paper would add substantially to the scope of this contribution. We found the experts' reviews very thorough and thoughtful and agree with their comments. We all agree that your manuscript addresses an important question: considering the continuity and non-linearity of soil moisture effects on plant morpho-physiological traits. We also found this manuscript quite valuable and would be inclined towards recommending it after consideration of comments by the authors. We hope that you will find our feedback useful to revise your manuscript.

Here are additional points that we identified.

1. One motivation of the paper is to assess the benefit of considering the continuous variation of soil moisture when evaluating the environmental response of the phenotype. Your point is that it brings a formally neglected layer of information that brings us one step closer to a more realistic assessment. I fully agree with this but I'm concerned about the presentation of the contextual background and the presentation of the implications of your work in the discussion. My concern comes from the fact that you do not "add up" a layer of detailed information to an otherwise realistic background. You rather proof test whether adding this environment as a continuous variable brings up useful information and you do this in an experimental simplified biological set up in order to properly proof test your hypothesis. Indeed, you use an experiment, a limited number of genetic lineages and summarise the continuous variation of the soil moisture to 6 modalities. This is not independent from another concern: It is sometimes clear, and sometimes less clear throughout the manuscript whether your contribution is specifically about "soil moisture" or whether you address a general issue that concerns all environmental variables. You want to revisit the text of the paper to clarify these aspects throughout the preprint so that no confusion is possible. It will bring value to the paper because your valuable contribution will be more clearly identified by readers as a result (and referred to). 2. An important implication of your work is that using environmental variables that are not continuous does not allow to observe nonlinear phenotypic responses. Missing such exponential or logarithmic responses has implications when predicting plant responses, in particular in the current context of climate changes. I totally agree with this and you could actually elaborate a bit more on this aspect and acknowledge more precisely similar work claiming the same message (eg work by Nussey, Wilson, Brommer who you cite, in wild populations, etc.).

3. You use quantitative genetic approaches and that brings value to this paper. However, the paper lacks contextual background about this aspect. You do not clearly state what are the methods used to assess continuous environmental effects (eg random regression quantitative genetic models) and whether they were used to assess environmental effects on plants. What are the differences and similarities with your approach (the aim is clearly the same)? 


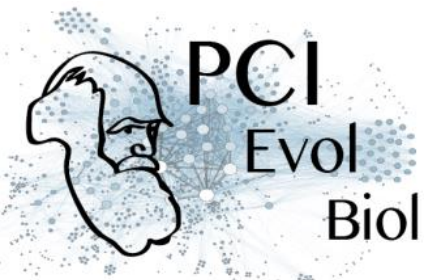

We would ultimately accept to recommend this preprint provided that you address these concerns and the reviewers' comments appropriately.

4. The contrasting patterns of evolutionary constraints between species are quite impressive. I wonder to what extent this contrasting pattern associates to the contrasting life-history strategies as the authors assume, or rather represent non-random phylogenetic signals unrelated to life-history strategies. While this is clearly beyond the focus of the manuscript it would be nice to have additional information on the strategies followed by other populations or sister species to give stronger support to the idea of life-history related genetic constraints.

Regards,

Benoit Pujol and Rodrigo Medel

Preprint DOI: $10.1101 / 2020.02 .17 .952853$

\section{Reviewed by anonymous reviewer, 2020-04-28 12:13}

Review of Monroe, Cai and Des Marais " Trait plasticity and covariance along a continuous soil moisture gradient"- PCl 2020

This manuscript analyses the response (variance and covariance) of 12 traits to continuously varying soil moisture gradient in five genotypes of two Brachypodium species (one annual and one perennial). The key findings are that i) most traits exhibit a non-linear response to drought leading to changes in trait correlations, and ii) the shape of the response varied between species, genotype and traits suggesting a context dependent response.

I really enjoyed reading this article. I agreed with the motivation of the article, the design, the code/data provided and the conclusion. Yet, I have to admit I am not a specialist of all the methods the authors used in this manuscript.

I still have some suggestions about the manuscript in its current form.

Introduction

Part of the discussion is about the difference between perennial and annual plants. It would be interesting to bring this subject in the introduction first. I haven't notice that one sp was annual and the other perennial before the discussion (while it is clearly mentioned by the authors in the material and methods). It would be interesting to get a paragraph in the end of introduction with some hypotheses about difference in traits variance, correlation and evolvability etc. between perennial and annual plants facing drought stress.

Material \& Methods

Plasticity through multidimensional trait space. - You specified that the traits are centered, but it is not clear to me if they are also reduced? I guess they are. Please clarify, this information impacts our interpretation of fig3.

Results

Non-linearity in trait responses to soil moisture. I would be interested to see the R2 of the best (final) model in Table 1, to have an idea of the part of the variance explained by each model. For each trait, if the R2 (or AIC) of the best models are very closed I would advise the author to use a model averaging approach (Grueber et al 2011) to get averaged estimates, and then predicted values based on these averaged estimates.

I guess the authors plotted in Fig 2 the predicted value for the traits at each soil moisture level, rather than the observed values. Please specify. 


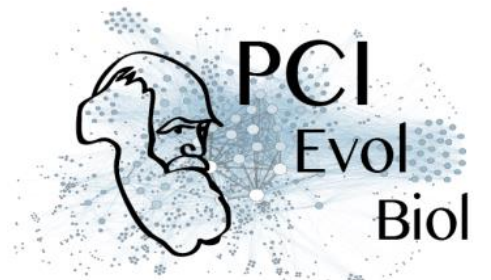

In the same line of thought, I think it would be interesting to provide the confident interval around the values (whenever they are predicted or fitted values) plotted in fig 2. Maybe all the differences observed have overlapping $\mathrm{Cl}$, and are not significantly different all along the moisture gradient.

Cohort effect- I would be interested to see some discussion or interpretation of the significance of cohort effect. Indeed in Table1, the harvesting day $(\mathrm{H})$ which is the cohort effect is significant in 18 models on 24 . Do you have any explanation? Is it due to a change in trait value with plant age? Or is it an artefact of the experimental design? As you already know, plant traits can shift with plant ontogeny. In one hand, we can think that it is unlikely to see this kind of effect between 5days-difference Brachypodium plants. On the other hand, five days differences for a plant of 43 or 56 days is not negligible ( $9-11 \%$ of its lifetime). Please discuss this result.

I found the Fig3 $b$ and c complicated to understand, and I am not sure they provide a lot of information to the reader. In my understanding the authors only get one point form them: phenotypes were similar between extreme dry and extreme wet soil moisture contents for B sylvaticum. I have to admit that this result was not particularly obvious for me on fig3b and c. Maybe the authors should consider dropping Fig 3 part b and $c$ into appendix to simplify their ms.

Fig4- So each point is a correlation coefficient calculated across 5 genotypes, right? If yes please specify this in the legend. If No, please add the info about the sample size used to drawn each correlation as different point size in the plot.

\section{Discussion}

Just a general comment/question to the authors. I agree that the next step would be to search for general patterns of variation and covariation across species (which is a goal of functional ecology) along discrete environmental variables. As you specified, the best approach is to reproduce in an analogous fashion the same experiment on more genotypes or other species. Yet, do you think we could get some insights by compiling individual studies focusing on discrete soil water levels? Like a meta-analyses or a trait database with information on the soil water content (and type of soil)?

Minor comments:

- In Abstract please replace "multiple genotypes" by "five genotypes"

- In Material \& Methods section- Genotypes and species: "For each species, these genotypes represent a range of geographical origin and phenotypic diversity" Please specify into bracket the name of two most distant locations. It will give some clue to the reader without having to go through your previous work. - In fig 3, please add in legend "B sylvaticum is colored in orange and B.distachyon blue".

- In section Non-linearity in trait responses to soil moisture is pervasive. "In contrast, SLA, total biomass and shoot mass did not include environmental predcitor" please replace "predcitor" by "predictor"

- In discussion please check for references in the text. For instance you did not provide the year of publication for Lundgren and Des Marais (current biology).

- In appendix, I would be useful to provide a schema of the experimental design, with information about the number of genotypes, cohort, traits measurement along the five days etc.

Grueber et al 2011 - Methods in Ecology and Evolution. Multimodel inference in ecology and evolution: challenges and solutions https://doi.org/10.1111/j.1420-9101.2010.02210.x

\section{Reviewed by anonymous reviewer, 2020-05-19 10:36}

The scope of the manuscript is to understand the phenotypic response of genotypes to an environmental gradient of drought, with the goal to better predict the shape and form of phenotypes and response to selection. The authors argue that so far, we have studied phenotypic responses (to abiotic factors) in discrete experimental setups, and it is about time to integrate the complexity of continuous environmental variation in experimental designs so that we can predict phenotypic responses and selection in a more "realistic" way, 


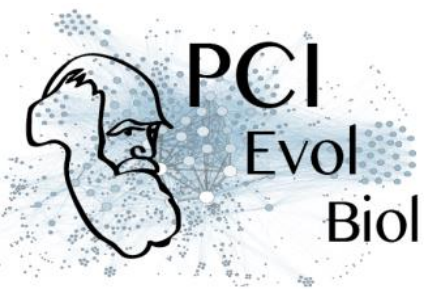

as opposed to a set of fixed levels. I agree with the authors that experiments under controlled conditions often use a number of fixed levels and measure the response of phenotypes, and we need to understand better the covariation between phenotypes and environmental gradients to estimate selection and the response to selection more accurately. But I disagree with the statement that we have not studied much continuous variation, or that we don't have tools for that. This has been done quite extensively on phenotypic selection studies. The work of Lande \& Arnold (1983) Evolution 37: 1210-1226 and subsequent methodological papers in the context of phenotypic selection have provided the methods to understand the covariation between traits and fitness to measure selection on phenotypic traits while controlling by genetic correlations (or phenotypic as a proxy of genetic correlations), including non-linear relationships.

Furthermore, the approach to model phenotypic traits as a function of environmental traits through model selection has been already used in phenotypic selection studies (e.g. Bolstad et al (2010) New Phytologist 188:370-384, use of model selection to creating a fitness function based on functional traits). Perhaps we have simply failed to quantify environmental factors in natural conditions to better predict the correlation between traits and continuous environmental factors. But after reading the manuscript, I am left with mixed feelings about the adequacy of their methods. Yes, with their experiment the authors create environmental gradients to measure the reaction norm of genotypes to soil moisture, but this is done in only 5 genotypes per species. And I am not surprised: the experimental design is way too complicated and large when one adds more genotypes. I guess this is why there's been a tendency of using modest and simple experimental approaches where we can increase our sample size of genotypes per species to study the evolution of phenotypes and what constrains a phenotypic response to selection. While the authors used a smart experimental and sophisticated statistical way to investigate the relationship between continuous environmental variation and phenotypes, they are constrained by the scale of the experiment. So in summary, either we make a simple design (fewer levels) and increase the range of genotypes (and potentially phenotypes), or we focused on a subset of genotypes and we get to understand well reaction norms.

I like how the authors approach and recognized the importance of studying specific traits, the structure of genetic correlations and covariation between traits, but also the general multivariate perspective. I admit that the phenotypic plasticity in a multivariate fashion is hard to grasp but necessary. But I was somewhat surprised to see that, while they emphasize the importance of the multivariate approach, little was brought into the discussion, and the focused was on specific traits that somehow "made sense" in the context of lifehistory traits. So perhaps the authors should think of improving the alignment between the introduction and discussion. Furthermore, I missed a more ecological and biological context in the introduction to predict responses of the species under study, which is possible since this is a model organism. The life history trait argument and adaptation to specific environments in the context of the geographic range of the species is overlooked in the introduction, and yet it plays an important role to explain plasticity and evolvability in the two species. This is simply discussed in the context of annual and perennial life history strategy and adaptation to drought. But the results are important to predict how will the two species respond to environmental stress through drought and drought tolerance. This is where their experimental manipulation is useful and it could enrich the discussion: what are the implications of their estimates to predict an evolutionary response to an increase in drought? What species will be more constrained and how from a multivariate and single trait approach? What would be the line of least resistance for phenotypic evolution to occur in both species? These seem to me the interesting questions, particularly in the context of their 4th paragraph in the introduction (see below). However, the study includes only 5 genotypes per species and I would assume that this represents only a small fraction of the genetic and phenotypic variation of the two species, and a small fraction of the phenotypic responses if local adaptation and population differentiation is important. This should be, at the very least, acknowledged in the discussion.

The 4th paragraph of the introduction is beautifully presented and argued, and I feel that the authors have put forward an interesting theoretical context as to why is it important to investigate the structure of variance and covariance of traits in environmental gradient to predict phenotypic responses and how traits evolve. I think it is important to return to these ideas in the discussion and use their results to solve the conundrum presented (see above). 
From a methodological perspective, the study is well executed and the authors displayed a wide collection of statistical tools to investigate phenotypic responses from a multivariate fashion. However, there are a few flaws in the Methods making necessary much reading between the lines. More detailed is needed to add clarity so that the authors can reach a wider audience, for example:

- In the experiment, the authors stated "For each genotype, 1200 were planted..." but the experimental setting of Figure S2 doesn't match this. Based on that figure, it looks like the authors have a block design where they used all genotypes $x 6$ irrigation treatments $x 5$ replicates, replicating each genotype per block to ensure they can measure all traits. It took a while to figure this out. So there are a total of 10 plants per genotype and treatment, not 100 as it sounded from the initial description. Unless I'm wrong. Please clarify. - I am confused with the soil moisture estimate, as it appears to be a function of multiple measures (pot weight in day $d$, weighed dry soil, and field capacity). But if pot weight is used to estimate soil moisture, moisture is somehow influenced by how genotypes used hydric resources right? In other words, they predict traits as a function of how hydric resources are consumed under different drought regimes right?

- Use of model selection to predict traits as a function of genotype, linear, and non-linear soil moisture. It would be good to provide more details on model selection. In general, people usually report AIC values of models to show what's the best model. It is then confusing to also add $p$ values, as opposed to the coefficients used to build the function to predict the trait, which by the way would be useful for other authors. Those coefficients are actually more informative than $p$ values. I suppose that it would be impractical in terms of an appendix to add details of model selection for every single trait.

- The methods on plasticity through multidimensional trait space are quite obscure, please provide more details as much reading between the lines is necessary to follow this section.

The results are not easy to follow so it would be good to add clarity. I have picked up a few issues:

2nd paragraph: Be consistent with the use of acronyms in the text. For example, (...) in leaf relative water content (RWC). This will facilitate your readership to follow the storyline.

Figure 2 represents variation in phenotypic responses ranging between $25 \%$ to $100 \%$, and the $10 \%$ tail is not represented. Also, make sure that you specify the color code in all your figures

3rd paragraph, description of non-linearity in trait responses: for a few traits, the best model based on AIC does not include the $\mathrm{E}$ term, although not all the traits in that situation are included in the description of the results, and it is unclear why do you add the $E$ term in models when it does not seem to be important to predict some traits. I am also confused with the results when looking at Table 1 and Figure 2, e.g. C content in B. sylvaticum does not seem to be predicted by any non-linear term, and yet figure $2 \mathrm{~h}$ shows a non-linear quadratic response, stronger than other traits with a detected quadratic response according to model selection, or even for $\mathrm{B}$. distachyon with a model with quadratic $\mathrm{E}$ terms. This does not make sense and perhaps the authors should revise their models. This happens for several other traits. It is also unclear how you used model selection. You seem to use both AIC of the model and $p$ values per term in your model, some of which are not statistically significant $(p>0.05)$ and I would imagine that in those cases including or including not those terms would not make any difference (as based on AIC values). So please clarify the logic to create the traits as a function of parameters, because not always you seem to use the best model as defined by model selection.

4th paragraph, PCA results. I am somewhat confused here. Looking at figures $3 \mathrm{~b}$ and $3 \mathrm{c}$ it does not look as if B. sylvaticum (yellow) displayed similar phenotypes at the extreme of the gradients but in B. distachyon (blue). For the species coded in blue, it appears that response presents more variation in PC2 and 3 within genotype. So perhaps the authors need to improve the narrative of the explanation to better reflect the results of the PCA. It's unclear whether they refer to within genotypes or, in general, among genotypes.

5th paragraphs, results on significant interaction between genotype and non-linear predictors. It's confusing to use $\mathrm{p}$ values and model selection at the same time. In essence, with model selection you look at what model explains best the data collected, and so in some cases, the interaction term is important as it gives the model with the lowest AIC value. But most of the traits did not show that interaction effect term between $\mathrm{G} x$ 
E (be E either linear, quadratic or second degree natural spline parameter. I wonder whether that's a limitation of your experiment: you simply screen 5 genotypes per species.

I find that the discussion does not really answer the questions of the manuscript. The scope is to study plasticity and covariance along an environmental gradient, but this does not seem to be discussed well. The core of the manuscript is paragraph 4th of the introduction, and I was expecting a discussion around this. So yes, we learn that we can predict a trait as a function of environmental factors (with some limitations as to how many genotypes can we include), and that non-linear relationships are important. But the implications to predict how traits evolve and how phenotypes evolved are poorly discussed. The authors identified really interesting trends on plasticity and evolvability, with the two species showing opposite response to soil moisture, which somewhat seems to reflect life-history traits and the geographic and climatic conditions in which the two species evolved. So while the authors looked at specific traits, the discussion needs to develop more in the context of how phenotypes respond to the environmental gradient, from individual traits (somehow done) but also as complex phenotypes, otherwise the multivariate methods seem pointless. The paragraph on implications for breeding drought adaptation was not expected. I grant you that your work is important for crop breeding, but I don't think it deserves a paragraph. After all, no mention of crop breeding was done in your aims.

There are a few typos in the text (e.g. it's when it should be its, changes in verb tense) but I guess the authors can fix this in the new version. A few more comments have been added to the text. I might sound very critical in my review, but I really enjoyed your manuscript and it is inspirational.

End of review

Download the review (PDF file)

\section{Author's reply:}

Download author's reply (PDF file) 